\title{
Conductas sexuales de riesgo y uso del cibersexo Comparación entre diferentes perfiles de uso del cibersexo
}

\author{
Marta García Barba \\ barbam@uji.es \\ Juan Enrique Nebot García \\ junebot@uji.es \\ Beatriz Gil Juliá \\ bgil@uji.es \\ Cristina Giménez García \\ gimenezc@uji.es
}

\section{Resumen}

Introducción: El uso del cibersexo es una práctica sexual bastante extendida que, empleada de forma inadecuada, puede tener consecuencias negativas como facilitar prácticas sexuales de riesgo.

Objetivo: El objetivo de este estudio es comprobar si un uso abusivo del cibersexo influye en la frecuencia en la que se realizan prácticas sexuales de riesgo.

Método: Participaron un total de 160 personas ( 80 perfil recreativo y 80 perfil de riesgo de uso del cibersexo) con edades comprendidas entre los 18 y los 28 años $(M=22,36$; $D T=2,66)$. Todos completaron la versión española del Internet Sex Screening Test (ISST) (Ballester-Arnal, Gil-Llario, Gómez-Martínez y GilJuliá 2010) y algunas preguntas sobre prácticas sexuales de riesgo.

Resultados: No se encontraron diferencias significativas entre ambos grupos en cuanto a la frecuencia en la que mantienen relaciones sexuales. Existe una asociación positiva entre un mayor abuso del cibersexo y conductas de riesgo en sexo oral, anal, con pareja esporádica y tras haber consumido alcohol y otras drogas. El grupo que consume cibersexo de manera abusiva ha realizado más prácticas sexuales observadas en Internet, a pesar de saber que pueden ser peligrosas (como asfixia), que aquellos que realizan un uso recreativo de esta herramienta.

Conclusiones: Se presenta un patrón diferencial, en función del consumo de cibersexo, en prácticas sexuales de riesgo que exponen la salud física y mental de los jóvenes. Por ello, consideramos importante realizar actividades preventivas que informen sobre el uso de esta herramienta, sus beneficios e inconvenientes y cómo reducir los riesgos relacionados con la misma. 
Palabras clave: cibersexo, prácticas sexuales de riesgo, uso abusivo, uso recreativo, salud.

\section{Abstract}

Introduction: The use of cybersex is a widespread sexual practice that may have negative consequences when used inappropriately, such as facilitating risky sexual practices.

Objective: The objective of this study is to verify if an abusive use of cybersex influences the frequency in which sexual risk practices are performed.

Method: A total of 160 people participated ( 80 recreational profile and 80 cybersex risk profile) with ages between 18 and 28 years $(M=22.36$; $S D=2.66)$. All completed the Spanish version of the Internet Sex Screening Test (ISST) (Ballester-Arnal, Gil-Llario, Gómez-Martínez \& Gil-Juliá 2010) and some questions about risky sexual practices.

Results: No significant differences were found between both groups regarding the frequency in which they have sexual relations. There is a positive association between more abuse of cybersex and risk behaviors in oral sex, anal sex, with sporadic partner and after having consumed alcohol and other drugs. The group that consumes cybersex abusively has performed more sexual practices observed on the Internet, despite knowing that they can be dangerous (such as asphyxia), than those who make recreational use of this tool.

Conclusions: There would be a differential pattern, based on the consumption of cybersex, in risky sexual practices that expose the physical and mental health of young people. For this reason, we consider important to carry out preventive activities that inform about the use of this tool, its benefits and drawbacks and how to reduce the risks related to it.

Keywords: Cybersex, risky sexual practices, abusive use, recreational use, health.

\section{Introducción}

El cibersexo, definido como el uso de Internet con fines sexuales, es uno de los fenónemos emergentes relacionado con las tecnologías de la información y comunicación (TIC). Este concepto abarca todas aquellas actividades que se realizan a través de Internet con propósito sexual (Cooper y Griffin-Shelley 2002), por lo que se incluyen tanto las actividades que se realizan en solitario (visionado de imágenes/vídeos eróticos, etc.) como aquellas que requieren la implicación de otra persona (chats, videollamadas, etc.). El uso de esta herramienta se encuentra bastante extendido, siendo las actividades que se dan con más frecuencia aquellas que se realizan en solitario, como acceder a material sexual online estimulante $(76,5 \%)$; mientras que las que requieren interacción con otros usuarios son menos frecuentes (30,8 \%) (Döring, Daneback, Shaughnessy, Grov y Byers 2017).

Dependiendo de aspectos como la frecuencia de uso del cibersexo, el tiempo invertido y las consecuencias experimentadas, se definen tres perfiles de usuarios que irían en un continuo: usuarios recreativos (aquellos que realizan un uso moderado del cibersexo y que, en principio, no experimentarían consecuencias negativas), usuarios de riesgo (quienes empiezan a presentar algún síntoma de adicción, como una inversión de tiempo excesiva en el uso del cibersexo) y usuarios compulsivos sexuales 
online (Cooper, Putnam, Planchon y Boies 1999). Estos últimos presentan una relación con el cibersexo caracterizada por una alta frecuencia y falta de control, persistencia en la actividad a pesar de las consecuencias negativas e interferencia en distintos aspectos de la vida (Delmonico y Carnes 1999; Philaretou, Mahfouz y Allen 2005; Schneider 1994). Aparte de estos perfiles, Delmonico, Griffin y Moriarity (2001) añaden un cuarto perfil dentro de los usuarios recreativos para diferenciar a aquellos que hacen un uso apropiado, en el cual ensalzan su experiencia sexual y su sexualidad sin ocultarse y sin mostrar vergüenza, de aquellos que hacen un uso inapropiado.

Las personas que pertenecen al perfil de uso recreativo, a priori, no experimentan ninguna consecuencia negativa e incluso se benefician de los aspectos positivos que guarda esta actividad. Estos aspectos positivos incluyen la accesibilidad a información sobre sexualidad y sexo (Simon, Daneback y Ševčíková 2014), el establecimiento de nuevas relaciones (Leiblum y Doring 2002), el incremento de la satisfacción y el placer sexual (Daneback, Ševčíková, Mansson y Ross 2013), ser fuente de apoyo y confort respecto a la orientación sexual (Ross y Kauth 2002), mejorar las habilidades de comunicación interpersonal y mejorar las relaciones de pareja (Freeman-Longo, Brown y Price 2002; Tepper y Owens 2002). Sin embargo, cuando el consumo resulta más problemático, el cibersexo también cuenta con consecuencias negativas que se ven exacerbadas. Estas consecuencias son tales como la formación de actitudes y creencias sexuales disfuncionales, el modelado negativo de ciertas prácticas sexuales, comportamientos sexuales de riesgo (Tsitsika et al. 2009), dificultades para formar relaciones íntimas (Weinstein, Zolek, Babkin, Cohen y Lejoyeux 2015), la necesidad de buscar cada vez material que produzca mayores niveles de excitación debido a la saciación, el ciberacoso y, sobre todo, la posibilidad de desarrollar una adicción (Flood 2010).

Algunos estudios se han centrado en explorar aquellas características o aquellos factores que hacen que algunas personas sean más vulnerables a la hora de padecer este tipo de adicción. Estos factores son la timidez (Ross, Rosser, McCurdy y Feldman 2007), la baja autoestima, una imagen corporal distorsionada, una alta tendencia a la búsqueda de sensaciones sexuales (Luder et al. 2011), disfunciones sexuales no tratadas o una previa adicción sexual. Otros estudios han encontrado que el uso problemático de la actividad sexual online está asociado con una variedad de condiciones psicopatológicas comórbidas, incluyendo el abuso de sustancias, la presencia de otras adicciones, el trastorno de hiperactividad con déficit de atención, los trastornos del estado de ánimo y los trastornos de ansiedad (Castro-Calvo, BallesterArnal, Gil-Llario, Morell-Mengual y Salmerón 2014; Wéry y Billieux 2017). Otros factores como las disfunciones sexuales o la regulación del estado del ánimo podrían desempeñar un papel importante en el desarrollo y mantenimiento de la adicción (Wéry y Billieux 2016, 2017). Ross, Månsson y Daneback (2012) relacionaron tres tipos de motivaciones que podían predecir el uso problemático del cibersexo. Estas motivaciones fueron un interés especial en contenido pornográfico que solo está disponible online (por ejemplo, contenido parafílico) y lograr la relajación y satisfacción sexual. Puesto que una de las consecuencias negativas del consumo abusivo del cibersexo es el modelado de prácticas sexuales, en las que podemos incluir las conductas sexuales de riesgo (Buhi et al. 2013; Fernández-Dávila y Zaragoza-Lorca 2011), el objetivo de nuestro estudio es comprobar si el hecho de realizar un consumo abusivo del cibersexo se relaciona con la realización de prácticas sexuales de riesgo o, por el contrario, únicamente afecta el hecho de utilizar esta herramienta, independientemente del tipo de consumo que se haga. 


\section{Método}

\section{Participantes}

En este estudio participaron un total de 160 jóvenes. De estos 160 participantes, el $54,38 \%$ eran hombres y el $45,62 \%$ eran mujeres, con edades comprendidas entre los 18 y los 28 años $(M=22,51$; $D T=2,27)$. La distribución por edades y género era homogénea $\left(X^{2}=10, p=0,446\right)$.

En cuanto a la orientación sexual, el $73,75 \%$ del total se identificaron como heterosexuales, seguidos del $15 \%$ que lo hizo como homosexuales y el $11,25 \%$ restantes que se identificaron como bisexuales. Encontramos diferencias significativas entre hombres y mujeres respecto a la orientación sexual, siendo la bisexualidad la segunda orientación sexual más frecuente entre las mujeres $(16,44 \%)$ y la homosexualidad la segunda entre los hombres $(20,69 \%)\left(X^{2}=7,37, p=0,025\right)$.

Respecto al nivel académico, el 78,7 \% tenía estudios superiores (grado, licenciatura o máster) y el resto contaba con ciclos formativos (10\%), estudios de bachillerato (10\%) y graduado escolar $(1,3 \%)$.

\section{Instrumentos}

Los instrumentos empleados fueron dos: el Internet Sex Screening Test (ISST) de Delmonico (1997) en la adaptación española de Ballester-Arnal, Gil-Llario, GómezMartínez y Gil-Juliá (2010) y una escala desarrollada ad hoc sobre prácticas sexuales con 8 ítems que exploran la frecuencia de la conducta sexual y el uso del preservativo.

El cuestionario de adicción al cibersexo (ISST) es un instrumento empleado para medir la conducta sexual online y está compuesto por 25 ítems con opción de respuesta dicotómica (verdadero $=1 /$ falso $=0$ ). Las puntuaciones totales de esta escala oscilan entre 0 y 25. Esta escala permite discriminar entre diferentes perfiles de uso del cibersexo, diferenciando entre perfil de uso recreativo para aquellos que obtengan puntuaciones entre 0 y 8 , perfil de riesgo de adicción para los que puntúen entre 9 y 18 y perfil de adicción para aquellos con puntuaciones superiores a 18. La escala está compuesta por cinco factores: compulsividad sexual online (ítems $2,6,7,15,16,18$, 19 y 25), comportamiento online solitario no compulsivo (ítems $1,5,10,11,13$ y 14), comportamiento online social (ítems $8,9,20,22,23$ y 24), gasto económico online (ítems 3 y 4) y percepción de gravedad del comportamiento online (ítems 12, 17 y 21). Este cuestionario tiene una alta consistencia interna $(\alpha=0,88)$ y una buena validez convergente y discriminante (Ballester-Arnal, Gil-Llario, Gómez-Martínez y Gil-Juliá 2010).

La escala desarrollada ad hoc sobre conducta sexual y uso del preservativo cuenta con una pregunta que recoge la frecuencia del uso del preservativo (desde nunca hasta siempre, pasando por no lo he practicado) en diferentes prácticas y escenarios sexuales (sexo vaginal, anal, oral, relaciones con pareja estable, con pareja esporádica y tras haber consumido alcohol / otras drogas). Las puntuaciones en estos ítems oscilan entre 0 (conducta segura $=$ siempre se utiliza preservativo $/$ no se ha practicado) y 5 (conducta de alto riesgo = nunca se utiliza preservativo). También incluye otras preguntas respecto a la actividad sexual y su frecuencia en la actualidad, al uso de Internet con fines sexuales y al haber realizado prácticas sexuales de riesgo vistas en Internet. 


\section{Procedimiento}

La información del estudio se difundió a través de las redes sociales, en un mensaje que contenía el enlace al cuestionario online. Una vez los participantes daban su consentimiento informado, procedían a cumplimentarlo de forma voluntaria y anónima.

Contamos con un total de 627 personas de las que finalmente participaron 160 de ellas, al cumplir con los diferentes criterios de inclusión. Estos criterios de inclusión fueron tener entre 18 y 28 años, contar con competencias lingüísticas suficientes para comprender el castellano y haber respondido la totalidad del cuestionario. La población se dividió en dos grupos según la puntuación obtenida en el ISST: riesgo de adicción y no riesgo/uso recreativo. En función del perfil de usuario de cibersexo, el $50 \%$ de las personas participantes se identificó en situación de riesgo de adicción y el otro $50 \%$ como usuario recreativo.

Los análisis estadísticos se realizaron mediante el programa estadístico SPSS versión 25.0. Por un lado, se realizaron pruebas de chi cuadrado con el interés de conocer posibles diferencias en la frecuencia de las relaciones sexuales y las prácticas de riesgo entre ambos grupos, dada la importancia que la frecuencia de las conductas puede tener para la exposición ante las infecciones de transmisión sexual (ITS). Además, se llevó a cabo la correlación de Spearman empleada para explorar una posible asociación entre el riesgo en las relaciones sexuales y las puntuaciones del uso problemático del cibersexo.

\section{Resultados}

Inicialmente se quiso comprobar si ambos grupos diferían en cuanto a su actividad sexual offline. Como vemos en la figura 1 , no se encontraron diferencias significativas respecto a la existencia de actividad sexual entre las personas que se encuentran en el perfil de uso recreativo y las que se encuentran dentro del perfil de riesgo de adicción. En ambos casos, la mayoría mantienen o han mantenido relaciones sexuales en los últimos 3 meses $\left(x^{2}=0,36 ; p=0,548\right)$. Además, respecto a la frecuencia de la actividad sexual, más de la mitad de los encuestados de ambos perfiles mantienen relaciones sexuales entre 1 y más de 3 veces a la semana $\left(X^{2}=8,17 ; p=0,226\right)$. 


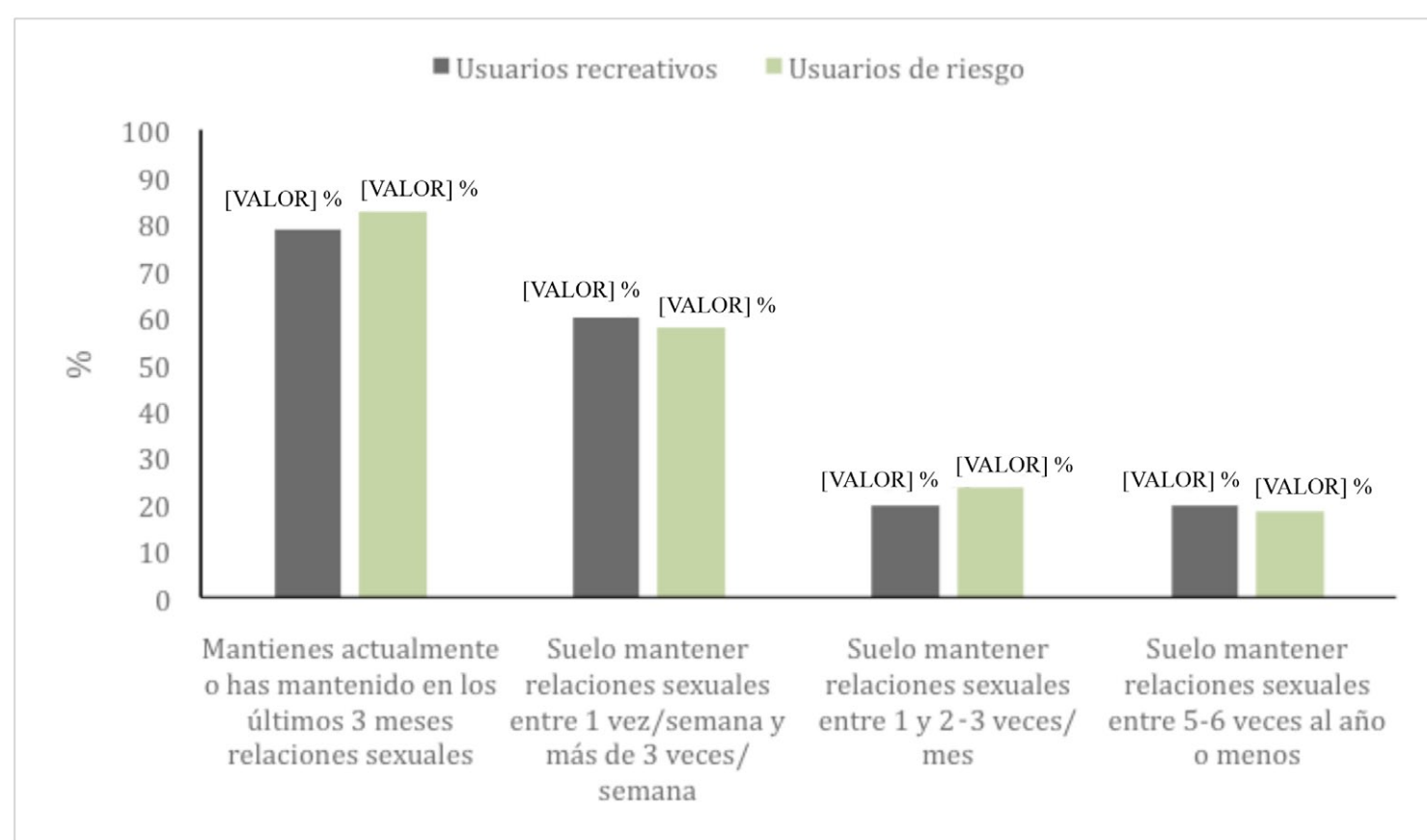

Figura 1. Diferencias en la frecuencia de la actividad sexual en función del perfil de uso del cibersexo.

A continuación, y centrándonos en el objetivo principal de esta investigación, se compararon las respuestas de ambos grupos respecto a la frecuencia del uso del preservativo en diferentes prácticas sexuales. Para ello, se dicotomizaron las respuestas a los ítems en función de si implicaban riesgo de transmisión de ITS (uso no sistemático del preservativo) y las que no implicaban riesgo de transmisión (uso sistemático del preservativo o nunca lo he practicado). Como vemos en la tabla 1 , son los usuarios de riesgo quienes realizan estas prácticas sexuales inseguras en mayor medida que los usuarios recreativos, a excepción del sexo vaginal. Además, estas diferencias son significativas en el sexo anal de riesgo, sexo de riesgo con pareja esporádica y sexo de riesgo tras consumo de alcohol / otras drogas.

Tabla 1

Análisis diferenciales de las prácticas sexuales de riesgo según perfil de uso de cibersexo.

\begin{tabular}{ccccc}
\hline & $\begin{array}{c}\text { Usuarios recreativos } \\
(\%)\end{array}$ & $\begin{array}{c}\text { Usuarios de riesgo } \\
(\%)\end{array}$ & $X^{2}$ & $p$ \\
\hline Sexo vaginal de riesgo & 61,25 & 60 & 0,03 & 0,871 \\
Sexo oral de riesgo & 93,75 & 98,75 & 2,77 & 0,096 \\
Sexo anal de riesgo & 47,50 & 75 & 12,75 & $\mathbf{0 , 0 0 1}$ \\
Sexo de riesgo con pareja estable & 68,75 & 77,50 & 1,56 & 0,212 \\
$\quad$ Sexo de riesgo con pareja & 40 & 65 & 10,03 & $\mathbf{0 , 0 0 2}$ \\
$\quad \begin{array}{c}\text { esporádica } \\
\text { Sexo de riesgo tras consumo de }\end{array}$ & 43,75 & 75 & 16,19 & $\mathbf{0 , 0 0 1}$ \\
alcohol / otras drogas & & & & \\
\hline
\end{tabular}

Seguidamente, se quiso comprobar si existía relación lineal entre el consumo abusivo del cibersexo y la realización de prácticas sexuales de riesgo, teniendo en cuenta que se evalúa el uso del cibersexo en un continuo y no de forma categorial, aunque la 
escala nos permita clasificar a los sujetos en diferentes perfiles. Tal y como se muestra en la tabla 2, existe una asociación positiva entre ambos, siendo estas significativas en el sexo oral, sexo con pareja esporádica y sexo tras haber consumido alcohol / otras drogas.

Tabla 2

Correlaciones entre el uso del cibersexo y diferentes prácticas sexuales de riesgo.

\begin{tabular}{|c|c|}
\hline & Uso de riesgo de cibersexo \\
\hline Sexo vaginal de riesgo & $r h o=-0,130 ; p=0,102$ \\
\hline Sexo oral de riesgo & $r h o=0,160 ; p=0,043$ \\
\hline Sexo anal de riesgo & $r h o=0,129 ; p=0,104$ \\
\hline Sexo de riesgo con pareja estable & $r h o=-0,023 ; p=0,775$ \\
\hline Sexo de riesgo con pareja esporádica & $r h o=0,227 ; p=0,004$ \\
\hline $\begin{array}{c}\text { Sexo de riesgo tras haber consumido } \\
\text { alcohol / otras drogas }\end{array}$ & $r h o=0,231 ; p=0,003$ \\
\hline
\end{tabular}

Finalmente, se comprobó que existían diferencias significativas en cuanto a la realización de prácticas sexuales de riesgo offline vistas a través de Internet $\left(x^{2}=12,91 ; p<0,001\right)$, siendo el grupo de riesgo de adicción al cibersexo quienes habían realizado estas prácticas en mayor medida $(38,75 \%)$ que las personas que presentan un perfil de uso recreativo del cibersexo $(13,75 \%)$.

\section{Discusión y conclusiones}

Como se ha podido observar a lo largo de estas líneas, pese a que se puede pensar que el uso del cibersexo es una alternativa para satisfacer el deseo sexual cuando no se puede satisfacer con una persona física (Ballester-Arnal, Castro-Calvo, Gil-Llario y Giménez-García 2014), nuestros resultados apuntan que las personas que se encuentran dentro del perfil de riesgo no difieren de las personas con un perfil de uso recreativo en cuanto a su actividad sexual offline. Ševčíková y Konečný (2011) encontraron en su investigación con adolescentes una relación positiva entre haber tenido experiencias sexuales offline y el uso de Internet con fines sexuales. Los adolescentes sexualmente experimentados eran más propensos a usar Internet con fines sexuales que los sexualmente inexpertos. Por lo tanto, quizás la relación se encuentre entre aquellos que estén más abiertos a explorar su sexualidad y el uso del cibersexo.

Por otra parte, y en la misma línea que los hallazgos obtenidos por Buhi et al. (2013) y Fernández-Dávila y Zaragoza-Lorca (2011), existe una relación positiva entre el uso de Internet y las prácticas sexuales de riesgo. Según nuestros hallazgos, las personas que se encuentran dentro del perfil de abuso del cibersexo realizan, en mayor medida, prácticas sexuales de riesgo, sobre todo cuando se pregunta por sexo anal, sexo con pareja esporádica y sexo tras consumo de alcohol / otras drogas. Estos resultados tienen especial relevancia ya que estamos hablando de sexo anal, la práctica sexual con mayor riesgo para la transmisión de $\mathrm{VIH}$, y las relaciones con parejas esporádicas y tras haber consumido alcohol / otras drogas, factor que se ha visto relacionado con un mayor número de prácticas sexuales de riesgo (Parkes, Wight, Henderson y Graham 2007; Scott-Sheldon et al. 2016).

Estos hallazgos nos indican que el uso abusivo del cibersexo puede estar implicado en las prácticas sexuales de riesgo, sobre todo en aquellas en las que existe un mayor riesgo de contraer una ITS. Por ello, se deberían realizar intervenciones preventivas 
dirigidas, principalmente, a enseñar cómo se hace un buen uso de esta herramienta y cómo ser críticos y analizar el contenido al que accedemos. Otras variables, como la búsqueda de sensaciones sexuales y la compulsividad, inciden en el uso de riesgo, por lo que cabría tener en cuenta la intersección de todas ellas en las intervenciones.

Este estudio cuenta con ciertas limitaciones que deberían tenerse en cuenta en el análisis de los resultados. En primer lugar, se trata de una muestra reducida, lo que impide que estos resultados puedan generalizarse. Además, dadas las desiguales prevalencias que en la población general existen entre usuarios recreativos, de riesgo y con adicción al cibersexo, en este estudio únicamente se han podido comparar dos de los tres perfiles de uso del cibersexo (recreativo y de riesgo). Por ello, consideramos que sería interesante incluir en un estudio posterior los tres tipos de perfiles para observar las diferencias en cuanto a la conducta sexual entre cada perfil de uso. Finalmente, destacamos que sería importante explorar tanto la conducta sexual como el uso del preservativo, así como los factores de personalidad subyacentes, para poder realizar un estudio más completo sobre la problemática tratada y avanzar más en su conocimiento para desarrollar intervenciones preventivas adecuadas.

\section{Referencias bibliográficas}

Ballester-Arnal, Rafael, Jesús Castro-Calvo, María Dolores Gil-Llario y Cristina Giménez-García. 2014. "Relationship status as an influence on cybersex activity: Cybersex, youth, and steady partner». Journal of sex \& marital therapy 40(5): 444-456

Ballester-Arnal, Rafael, María Dolores Gil-Llario, Sandra Gómez-Martínez y Beatriz GilJuliá. 2010. «Propiedades psicométricas de un instrumento de evaluación de la adicción al cibersexo». Psicothema 22(4): 1048-1053.

Buhi, Eric R., Natalie Klinkenberger, Mary McFarlane, Rachel Kachur, Ellen M. Daley, Julie Baldwin, Heather D. Blunt, Shana Hughes, Christopher Wheldon y Cornelis Rietmeijer. 2013. "Evaluating the Internet as a sexually transmitted disease risk environment for teens: findings from the communication, health, and teens study». Sexually transmitted diseases 40(7): 528-533.

Castro-Calvo, Jesús, Rafael Ballester-Arnal, María Dolores Gil-Llario, Vicente MorellMengual y Pedro Salmerón. 2014. «Internet, cibersexo y consumo de alcohol: estudio preliminar en adolescentes. International Journal of Developmental and Educational Psychology». Revista INFAD de Psicología 1(1): 507-516.

Cooper, Al y Eric Griffin-Shelley. 2002. «Introduction. The internet: The next sexual revolution». En Sex \& the internet: A guidebook for clinicians, ed. Alvin Cooper (1-15). New York: Brunner Routledge.

Cooper, Al, Dana E. Putnam, Lynn A. Planchon y Sylvain C. Boies. 1999. "Online sexual compulsivity: Getting tangled in the net». Sexual Addiction \& Compulsivity: The Journal of Treatment and Prevention 6(2): 79-104.

Daneback, Kristian, Anna Ševčíková, Swen-Axel Månsson y Michael W. Ross. 2013. "Outcomes of using the internet for sexual purposes: fulfilment of sexual desires». Sexual Health 10(1): 26-31.

Delmonico, David L. y Patrick Carnes. 1999. "Virtual sex addiction: When cybersex becomes the drug of choice». Cyberpsychology and Behavior 2(5): 457-463.

Delmonico, David L., Elizabeth Griffin y Joseph Moriarity. 2001. Cybersex unhooked: $A$ workbook for breaking free of compulsive online sexual behavior. Wickenburg, AZ: Gentle Path Press. 
Döring, Nicola, Kristian Daneback, Krystelle Shaughnessy, Christian Grov y E. Sandra Byers. 2017. "Online sexual activity experiences among college students: A four-country comparison». Archives of sexual behavior 46(6): 1641-1652.

Fernández-Dávila, Percy y Kati Zaragoza-Lorca. 2011. "Trust and sexual interaction: the significance of the Internet on the sex life and sexual risk behaviors of gay and bisexual men in Spain». International Journal of Sexual Health 23(2): 120138.

Flood, Michael. 2010. "The harms of pornography exposure among children and young people». Child Abuse Review 18: 384-400.

Freeman-Longo, Robert E., Seven M. Brown y Deborah Price. 2002. «Effects of internet sexuality on children and adolescents». En Sex \& the Internet: $A$ guidebook for clinicians, ed. Al Cooper (87-105). New York: Brunner Routledge.

Leiblum, Sandra y Nicola Döring. 2002. «Internet sexuality: Known risks and fresh chances for women». En Sex \& the Internet: A guidebook for clinicians, ed. Al Cooper (19-45). New York: Brunner Routledge.

Luder, Marie Thérèse, Isabelle Pittet, André Berchtold, Christina Akré, Pierre-André Michaud y Joan Carles Surís. 2011. "Associations between online pornography and sexual behavior among adolescents: Myth or reality?». Archives of sexual behavior 40(5): 1027-1035.

Parkes, Alison, Daniel Wight, Marion Henderson y Graham Hart. 2007. «Explaining Associations between Adolescent Substance Use and Condom Use». Journal of Adolescent Health 40(2): 180.e1.

Philaretou, Andreas, Ahmed Mahfouz y Katherine Allen. 2005. «Use of Internet pornography and men's well-being». International Journal of Men's Health 4(2): 149-169.

Ross, Michael W., Sven-Axel Månsson y Kristian Daneback. 2012. «Prevalence, severity, and correlates of problematic sexual Internet use in Swedish men and women ». Archives of Sexual Behavior 41(2): 459-466.

Ross, Michael W. y Michael R. Kauth. 2002. "Men who have sex with men, and the Internet: Emerging clinical issues and their management». En Sex and the Internet: A guidebook for clinicians, ed. Al Cooper (47-69).

Ross, Michael W., Brian R. Simon Rosser, Sheryl McCurdy y Jamie Lynn Feldman. 2007. "The advantages and limitations of seeking sex online: a comparison of reasons given for online and offline sexual liaisons by men who have sex with men». Journal of Sex Research 44(1): 59-71.

Schneider, Jennifer P. 1994. «Sex addiction: Controversy within mainstream addiction medicine, diagnosis based on the DSM-III-R and physician case histories". Sexual Addiction \& Compulsivity: Journal of Treatment and Prevention 1(1): 1944.

Scott-Sheldon, Lori A., Kate B. Carey, Karlene Cunningham, Blair T. Johnson, Michael P. Carey y MASH Research Team. 2016. "Alcohol use predicts sexual decision-making: a systematic review and meta-analysis of the experimental literature». AIDS and Behavior 20(1): 19-39

Ševčíková, Anna y Štephan Konečný. 2011. «An exploration of the relationship between real-world sexual experience and online sexual activity among 17 year old adolescents». Cyberpsychology: Journal of Psychosocial Research on Cyberspace 5(1): article $3 . \quad$ Recuperado de: https://cyberpsychology.eu/article/view/4244/3290 [Consultado el 4 de agosto de 2019].

Simon, Laura E., Kristian Daneback y Anna Ševčíková. 2014. «The Educational Dimension of Pornography: Adolescents' Use of New Media for Sexual Purposes». En Living in the digital age: self-presentation, networking, playing, and participating in politics, ed. Pascaline Lorentz, David Šmahel, Monika Metyková y Michelle Wright (33-48). Brno: Masaryk University. 
Tepper, Mitchell S. y Annette F. Owens. 2002. «Access to pleasure: Onramp to specific information on disability, illness, and changes throughout the lifespan». En Sex \& the Internet: A guidebook for clinicians, ed. Al Coopera (71-86). New York: Brunner Routledge.

Tsitsika, Artemis, Elena Critselis, Georgios Kormas, Eleftheria Konstantoulaki, Andreas Constantopoulos y Dimitrios Kafetzis. 2009. «Adolescent pornographic Internet site use: A multivariate regression analysis of the predictive factors of use and psychosocial implications ». CyberPsychology and Behavior 12(5): 545-550.

Weinstein, Aviv M., Rinat Zolek, Anna Babkin, Koby Cohen y Michel Lejoyeux. 2015. «Factors predicting cybersex use and difficulties in forming intimate relationships among male and female users of cybersex». Frontiers in psychiatry 6(54): 1-8.

Wéry, Aline y Joël Billieux. 2016. «Online sexual activities: An exploratory study of problematic and non-problematic usage patterns in a sample of men». Computers in Human Behavior 56: 257-266.

—. 2017. "Problematic cybersex: Conceptualization, assessment, and treatment». Addictive behaviors 64: 238-246. 